\title{
A RELATIONAL DESCRIPTION OF HIGHER COMMUTATORS IN MAL'CEV VARIETIES
}

\author{
JAKUB OPRŠAL
}

\begin{abstract}
We give a relational description of higher commutator operators, which were introduced by Bulatov, in varieties with a Mal'cev term. Furthermore, we use this result to prove that for every algebra with a Mal'cev term there exists a largest clone containing the Mal'cev operation and having the same congruence lattice and the same higher commutator operators as the original algebra. We also give a local variant of this theorem.
\end{abstract}

\section{INTRODUCTION}

Two algebras are called polynomially equivalent if they have the same underlying set and the same clone of all polynomial operations. One of the invariants to distinguish polynomially inequivalent algebras is the congruence lattice of the corresponding algebra, and the binary commutator operation $[\cdot, \cdot]$ on this lattice (the theory describing this commutator have been developed in the 80's, and is described in the book by Freese and McKenzie [8]). In fact, from the results of Idziak [10] and Bulatov [6], one can see that on the three-element set, every Mal'cev algebra is up to polynomial equivalence described by its congruence lattice, and the binary commutator operation. This is no longer true for sets with at least four elements. But one can generalize the binary commutator operator to higher arities. These higher arity commutators have been introduced by Bulatov [5]. From the description of polynomial clones with a Mal'cev operation on the four-element set [7, one can obtain that every four-element Mal'cev algebra is determined up to polynomial equivalence by its unary polynomials, congruence lattice, and higher commutator operators on this lattice. The higher commutators are defined by the following 'term-condition'.

Definition 1.1 (Bulatov's higher commutator operators). Let $\alpha_{0}, \ldots, \alpha_{n-1}$, and $\gamma$ be congruences of some algebra $\mathbf{A}$. We say that $\alpha_{0}, \ldots, \alpha_{n-2}$ centralize $\alpha_{n-1}$ modulo $\gamma$ if for all tuples $\mathbf{a}_{i}, \mathbf{b}_{i}, i=0, \ldots, n-1$, and all terms $t$ of $\mathbf{A}$ such that

(1) $\mathbf{a}_{i} \neq \mathbf{b}_{i}$, but the corresponding entries are congruent modulo $\alpha_{i}$ for all $i \in\{0, \ldots, n-1\}$, and

(2) $t\left(\mathbf{x}_{0}, \ldots, \mathbf{x}_{n-2}, \mathbf{a}_{n-1}\right) \equiv_{\gamma} t\left(\mathbf{x}_{0}, \ldots, \mathbf{x}_{n-2}, \mathbf{b}_{n-1}\right)$ for all $\left(\mathbf{x}_{0}, \ldots, \mathbf{x}_{n-2}\right) \in\left(\left\{\mathbf{a}_{0}, \mathbf{b}_{0}\right\} \times\right.$ $\left.\cdots \times\left\{\mathbf{a}_{n-2}, \mathbf{b}_{n-2}\right\}\right) \backslash\left\{\left(\mathbf{b}_{0}, \ldots, \mathbf{b}_{n-2}\right)\right\}$,

Date: July 11, 2018

2010 Mathematics Subject Classification. 08A40.

Key words and phrases. commutator theory, Mal'cev algebras, clone.

Supported by Austrian Science Fund (FWF), P24077: Algebraic approaches to the description of Mal'cev clones; Czech Science Foundation, GAČR 13-01832S; and Charles University in Prague, project SVV-2014-260107. 
we have

$$
t\left(\mathbf{b}_{0}, \ldots, \mathbf{b}_{n-2}, \mathbf{a}_{n-1}\right) \equiv_{\gamma} t\left(\mathbf{b}_{0}, \ldots, \mathbf{b}_{n-2}, \mathbf{b}_{n-1}\right) .
$$

The $n$-ary commutator $\left[\alpha_{0}, \ldots, \alpha_{n-1}\right]$ is then defined as the smallest congruence $\gamma$ such that $\alpha_{0}, \ldots, \alpha_{n-2}$ centralize $\alpha_{n-1}$ modulo $\gamma$. We define the nulary commutator to be trivially the full congruence on $\mathbf{A}$, and for the unary commutator of $\alpha$ we put $[\alpha]=\alpha$.

One of important notions that came from higher commutators is a notion of supernilpotence: an algebra is $k$-supernilpotent if it satisfies the commutator identity

$$
[\underbrace{1,1, \ldots, 1}_{k+1}]=0 \text {. }
$$

If an algebra is $k$-supernilpotent for some $k$ we say that is it supernilpotent. For general algebras supernilpotence is a strictly stronger notion then nilpotence; i.e., there is a nilpotent algebra which is not supernilpotent. However, this is not the case in the variety of groups where both notions coincide. Therefore supernilpotent algebras can be viewed as natural generalization of nilpotent groups. They also share several properties with nilpotent groups, in particular a Mal'cev algebra of finite type is supernilpotent if and only if it is a product of prime power order supernilpotent algebras [2]. It has been shown in 3] that there are two expansions of the same group that are both 2-supernilpotent, but the clone given as the join of their clones is not. In this paper we establish additional properties to ensure that the join of two $k$-supernilpotent clones sharing a Mal'cev operation is $k$-supernilpotent.

To achieve that goal we give a description of higher commutators using a certain $2^{n}$-ary relation denoted $\Delta\left(\alpha_{0}, \ldots, \alpha_{n-1}\right)$ (see Definition 3.1). A similar relation have been also defined in [13]. The relation $\Delta\left(\alpha_{0}, \ldots, \alpha_{n-1}\right)$ encodes the value of $\left[\alpha_{0}, \ldots, \alpha_{n-1}\right]$ as its forks at the last coordinate-by a fork of a relation $R$ at a coordinate $i$ we mean a pair $(a, b)$ such that there exists $\mathbf{c}, \mathbf{d} \in R$ with $c_{i}=a$, $d_{i}=b$, and $c_{j}=d_{j}$ for all $j \neq i$; and we denote $\psi_{i}(R)$, the set of all forks of $R$ at $i$. A similar notion has been used to investigate some properties of algebras with a cube term [4, 1]. The description of higher commutators is then given by the following theorem.

Theorem 1.2. If $\mathbf{A}$ is an algebra with a Mal'cev term, and $\alpha_{0}, \ldots, \alpha_{n-1}$ are congruences of $\mathbf{A}$ then

$$
\left[\alpha_{0}, \ldots, \alpha_{n-1}\right]=\psi_{2^{n}-1}\left(\Delta\left(\alpha_{0}, \ldots, \alpha_{n-1}\right)\right) .
$$

Further we show that $\Delta\left(\alpha_{0}, \ldots, \alpha_{n-1}\right)$ encodes not only the commutator $\left[\alpha_{0}, \ldots, \alpha_{n-1}\right]$ but also all smaller-arity commutators that can be obtained by omitting one or more of the congruences $\alpha_{i}$. We show that if we take the clone of all polymorphisms of the relation $\Delta\left(\alpha_{0}, \ldots, \alpha_{n-1}\right)$ we get exactly the clone $\mathcal{C}\left(\alpha_{0}, \ldots, \alpha_{n-1}\right)$ with the properties described in the following theorem, and consequently one can construct a largest clone with the same commutator operators as the original Mal'cev algebra.

Theorem 1.3. Let $\mathbf{A}$ be an algebra with Mal'cev term $q$, and let $\alpha_{0}, \ldots, \alpha_{n-1}$ be congruences of $\mathbf{A}$. Then there exists a largest clone $\mathcal{C}\left(\alpha_{0}, \ldots, \alpha_{n-1}\right)$ on $A$ containing $q$ such that it preserves congruences $\alpha_{0}, \ldots, \alpha_{n-1}$, and all commutators of the form $\left[\alpha_{i_{0}}, \ldots, \alpha_{i_{k-1}}\right]$ (where $k \leq n$ and $0 \leq i_{0}<\cdots<i_{k-1}<n$ ) agree in $\mathbf{A}$ and $\left(A, \mathcal{C}\left(\alpha_{0}, \ldots, \alpha_{n-1}\right)\right)$. 
Corollary 1.4. Let A be an algebra with a Mal'cev term $q$, then there exists a largest clone on A containing $q$ such that the algebra corresponding to this clone has the same congruence lattice as $\mathbf{A}$ and the same higher commutator operators as $\mathbf{A}$.

Proof of Corollary 1.4 given Theorem 1.3. The largest such clone is the intersection of all clones $\mathcal{C}\left(\alpha_{1}, \ldots, \alpha_{n}\right)$ from Theorem 1.3 for all $n$ and all tuples $\alpha_{1}, \ldots, \alpha_{n}$ of congruences of $\mathbf{A}$.

Although our main motivation of developing this theory lies in the application to Mal'cev algebras on a finite domain, the same results are valid even for algebras with infinite domains. Moreover, since the largest clone in the previous theorem is described as a polymorphism clone, we know that such clone is closed in the natural topology given by pointwise convergence by a result of Romov 12. More on clones on infinite sets can be found in $[9$.

The theory developed to prove Theorem 1.3 is strong enough to give relatively short proofs of several basic properties of higher commutators (usually referred as (HC1)-(HC8)) that have been established in [5], their proofs have been published in [2]. Our alternative proofs of some of these properties are given in the last section of this paper.

\section{Preliminaries and notation}

Algebras are denoted by bold letters, the underlying set of an algebra is denoted by the same letter in italic, Con $\mathbf{A}$ denotes the set of all congruences of an algebra $\mathbf{A}$, Clo $\mathbf{A}$ the set (clone) of all term operations of $\mathbf{A}, \operatorname{Cg} X$ denotes the congruence generated by $X, \operatorname{Sg} Y$ denotes a subalgebra generated by $Y$, and if $\alpha$ is a congruence then we use the symbol $a \equiv_{\alpha} b$ to denote $(a, b) \in \alpha$. Furthermore, if $R$ is a relation, we use symbol Pol $R$ to denote the clone of all polymorphisms of $R$. The symbol 2 will denote both the natural number 2 and the two-element set $\{0,1\}$.

We denote tuples by bold letters. The $i$-th coordinate of tuple $\mathbf{a}$ is denoted by either $a_{i}$, or $\mathbf{a}(i)$. So, $\mathbf{a}=\left(a_{0}, \ldots, a_{n-1}\right)$ and $\left(a_{0}, \ldots, a_{n-1}\right)(i)=a_{i}$. Tuples will be usually numbered by an increasing sequence of consecutive integers starting at 0 . So every $n$-ary relation is a subset of $A^{\{0, \ldots, n-1\}}=A^{n}$. The only exception will be elements of the relation $\Delta\left(\alpha_{0}, \ldots, \alpha_{n-1}\right)$. In the theory of binary commutator described in [8, it is usual to denote the elements of 4-ary relation $\Delta_{\alpha, \beta}$ (we will denote the same relation $\Delta(\alpha, \beta))$ as $2 \times 2$ matrices. Similarly, when generalizing this concept to $\Delta\left(\alpha_{0}, \ldots, \alpha_{n-1}\right)$ one should write elements of this relation as $2 \times \cdots \times 2$ $n$-dimensional matrices. We will denote those elements by tuples whose coordinates will be labeled by the set $2^{n}=\{0,1\}^{n}$, we will write these coordinates as binary sequences omitting brackets and commas, and if needed we will view them as reverse binary expansions of natural numbers $0, \ldots, 2^{n}-1$; i.e., the tuple $\mathbf{k}=k_{0} \ldots k_{n-1}$ represents the number $\sum k_{i} 2^{i}$. This gives us a natural linear ordering of the set $2^{n}$ that we will use to write the elements of $A^{2^{n}}$ as linear $2^{n}$-tuples. So, the tuple $\mathbf{a} \in A^{2^{n}}$ will be written as $\left(a_{00 \ldots 0}, a_{10 \ldots 0}, a_{010 \ldots 0}, a_{110 \ldots 0}, \ldots, a_{11 \ldots 1}\right)$. For $d \in\{0,1\}$ we will use symbol $\bar{d}$ for the negation of $d$, i.e., $\overline{0}=1$ and $\overline{1}=0$. We will also refer to forks of these relations at some coordinate $\mathbf{k}$ the same way as if all the coordinates would be integer.

The last piece of notation has a close connection to a simple lemma about forks of a relation. For any map $e: J \rightarrow I$ and $\mathbf{a} \in A^{I}$ the symbol $\mathbf{a}^{e}$ denotes the $J$-tuple 
defined by $\mathbf{a}^{e}(j)=a_{e(j)}$. Similarly, for a relation $R \leq \mathbf{A}^{I}, R^{e}$ denotes the relation $\left\{\mathbf{a}^{e} \mid \mathbf{a} \in R\right\}$.

Lemma 2.1. Let $\mathbf{A}$ be an algebra, $R \leq \mathbf{A}^{I}, S \leq \mathbf{A}^{J}, e: J \rightarrow I$, and $R^{e} \subseteq S$. If $i \in I$ and there is a unique $j \in J$ such that $e(j)=i$ then $\psi_{i}(R) \subseteq \psi_{j}(S)$. In particular,

(i) if $R \subseteq S$ then $\psi_{i}(R) \subseteq \psi_{i}(S)$ for every $i \in I$;

(ii) if $e: I \rightarrow I$ is bijective then $\psi_{e(i)}(R)=\psi_{i}\left(R^{e}\right)$ for every $i \in I$.

Proof. Suppose that $(a, b) \in \psi_{i}(R)$; i.e., there are tuples $\mathbf{a}, \mathbf{b} \in R$ such that $a_{i}=a$, $b_{i}=b$, and $a_{k}=b_{k}$ for all $k \neq i$. Then from $R^{e} \subseteq S$ we know that $\mathbf{a}^{e}, \mathbf{b}^{e} \in S$. These tuples witness that $(a, b) \in \psi_{j}(S)$, because $\mathbf{a}^{e}(j)=a_{i}=a, \mathbf{b}^{e}(j)=b_{i}=b$, and $\mathbf{a}^{e}(k)=\mathbf{b}^{e}(k)$ for $k \neq j$.

The statement (i) is a special case of the former for $I=J$, and $e$ being the identity mapping. To prove (ii), suppose that $e$ is a bijection on the set $I$. Then from the statement for $S=R^{e}$ we get that $\psi_{e(i)}(R) \subseteq \psi_{i}\left(R^{e}\right)$. For the other inclusion substitute $e$ with $e^{-1}, R$ with $R^{e}$, and $i$ with $e(i)$.

We recall two simple well-known lemmata for Mal'cev algebras.

Lemma 2.2. Let $\mathbf{A}$ be a Mal'cev algebra. Then any binary reflexive compatible relation on $\mathbf{A}$ is a congruence.

Lemma 2.3. Let $\mathbf{A}$ be a Mal'cev algebra, and let $R$ be $n$-ary compatible relation on $A$. If $(a, b) \in \psi_{i}(R)$, and $\left(c_{0}, \ldots, c_{i-1}, a, c_{i+1}, \ldots, c_{n-1}\right) \in R$ then

$$
\left(c_{0}, \ldots, c_{i-1}, b, c_{i+1}, \ldots, c_{n-1}\right) \in R .
$$

Proof. Without loss of generality suppose that $i=0$. Let $q$ be a Mal'cev term of $\mathbf{A}$, and let $\left(a, u_{1}, \ldots, u_{n-1}\right)$ and $\left(b, u_{1}, \ldots, u_{n-1}\right)$ be witnesses for $(a, b) \in \psi_{0}(R)$. Then

$$
q\left(\begin{array}{ccc}
b & a & a \\
u_{1} & u_{1} & c_{1} \\
\vdots & & \vdots \\
u_{n-1} & u_{n-1} & c_{n-1}
\end{array}\right)=\left(\begin{array}{c}
b \\
c_{1} \\
\vdots \\
c_{n-1}
\end{array}\right) \in R
$$

since we know that $R$ is compatible with $q$.

\section{Description of higher COMmutators}

Definition 3.1. Let $\mathbf{A}$ be an algebra, and $\alpha_{0}, \ldots, \alpha_{n-1} \in$ Con A. First, for each congruence $\alpha_{i}$ choose one dimension in the $n$-dimensional space. We define the relation $\Delta_{\mathbf{A}}\left(\alpha_{0}, \ldots, \alpha_{n-1}\right)$ as the $2^{n}$-ary relation indexed by the set $2^{n}$ generated by tuples that are constant on two opposing $(n-1)$-dimensional hyperfaces of the hypercube orthogonal to the dimension corresponding to $\alpha_{i}$ and these constants are $\alpha_{i}$ congruent.

We will use face ${ }_{i}^{d}(\mathbf{a})$ to denote the $(d+1)$-th hyperface orthogonal to dimension $i$, i.e., face $_{i}^{d}(\mathbf{a})=\mathbf{a}^{f_{i, d}}$ where $f_{i, d}(\mathbf{k})=k_{0} \ldots k_{i-1} d k_{i} \ldots k_{n-2}$. The generating tuples of the relation $\Delta_{\mathbf{A}}\left(\alpha_{0}, \ldots, \alpha_{n-1}\right)$ will be denoted cube $_{i}^{n}(a, b)$. By definition, face ${ }_{i}^{0} \operatorname{cube}_{i}^{n}(a, b)=(a, \ldots, a)$, and face ${ }_{i}^{1} \operatorname{cube}_{i}^{n}(a, b)=(b, \ldots, b)$; or equivalently, cube $_{i}^{n}(a, b)=(a, b)^{d_{i}}$ where $d_{i}: 2^{n} \rightarrow 2$ is defined by $\mathbf{k} \mapsto k_{(i)}$. Finally, $\Delta_{\mathbf{A}}\left(\alpha_{0}, \ldots, \alpha_{n-1}\right)$ is defined as

$$
\Delta_{\mathbf{A}}\left(\alpha_{0}, \ldots, \alpha_{n-1}\right):=\operatorname{Sg}\left\{\operatorname{cube}_{i}^{n}(a, b) \mid i<n, a \equiv_{\alpha_{i}} b\right\}=\bigvee_{i<n} \operatorname{cube}_{i}^{n}\left(\alpha_{i}\right) .
$$


For the trivial case when $n=0$, we put $\Delta_{\mathbf{A}}():=A$. If the algebra is clear from the context, we will write just $\Delta\left(\alpha_{0}, \ldots, \alpha_{n-1}\right)$ instead of $\Delta_{\mathbf{A}}\left(\alpha_{0}, \ldots, \alpha_{n-1}\right)$, and if $\mathcal{C}$ is a clone on the set $A$, we will write $\Delta_{\mathcal{C}}\left(\alpha_{0}, \ldots, \alpha_{n-1}\right)$ instead of $\Delta_{(A, \mathcal{C})}\left(\alpha_{0}, \ldots, \alpha_{n-1}\right)$.

Example. We will describe generators of $\Delta\left(\alpha_{0}, \alpha_{1}, \alpha_{2}\right)$ for three congruences $\alpha_{0}$, $\alpha_{1}, \alpha_{2}$ of an algebra $\mathbf{A}$. The elements of $\Delta\left(\alpha_{0}, \alpha_{1}, \alpha_{2}\right)$ are indexed by vertices of a three-dimensional hypercube. The generators are tuples of one of the following forms $(a, b, a, b, a, b, a, b)$, where $a \equiv_{\alpha_{0}} b,(a, a, b, b, a, a, b, b)$ where $a \equiv_{\alpha_{1}} b$, and $(a, a, a, a, b, b, b, b)$ where $a \equiv_{\alpha_{2}} b$. Their graphical representation is given in Figure 1 .
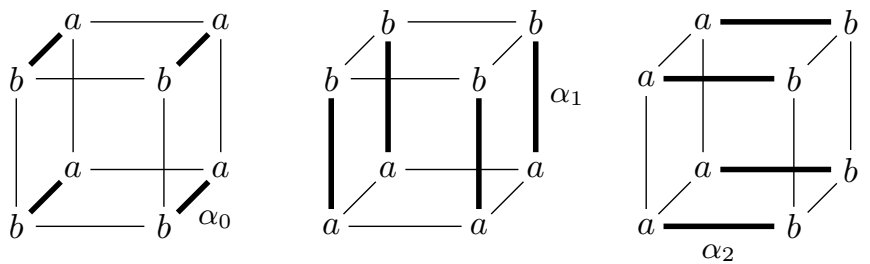

Figure 1. Generators of $\Delta\left(\alpha_{0}, \alpha_{1}, \alpha_{2}\right)$

Before we get to the proof of Theorem 1.2, we will describe some basic properties of the relation $\Delta\left(\alpha_{0}, \ldots, \alpha_{n-1}\right)$. The first lemma gives a term description of $\Delta\left(\alpha_{0}, \ldots, \alpha_{n-1}\right)$. This description gives a clear connection of $\Delta\left(\alpha_{0}, \ldots, \alpha_{n-1}\right)$ and the term condition.

Lemma 3.2. For every algebra $\mathbf{A}$, and congruences $\alpha_{i} \in \operatorname{Con} A, i<n$,

$$
\begin{aligned}
& \Delta\left(\alpha_{0}, \ldots, \alpha_{n-1}\right) \\
&=\left\{\left(t\left(\mathbf{a}_{0}, \ldots, \mathbf{a}_{n-1}\right), t\left(\mathbf{b}_{0}, \mathbf{a}_{1} \ldots, \mathbf{a}_{n-1}\right), \ldots, t\left(\mathbf{b}_{0}, \mathbf{b}_{1} \ldots, \mathbf{b}_{n-1}\right)\right) \mid\right. \\
&\left.\qquad i<n: m_{i} \in \mathbb{N}_{0}, \mathbf{a}_{i}, \mathbf{b}_{i} \in A^{m_{i}}, \mathbf{a}_{i} \equiv_{\alpha_{i}} \mathbf{b}_{i} ; t \in \operatorname{Clo}_{\sum m_{i}} \mathbf{A}\right\}
\end{aligned}
$$

where the elements of the $2^{n}$-tuple include the term $t$ applied to all the combinations of corresponding $\mathbf{a}_{i}$ 's and $\mathbf{b}_{i}$ 's.

Proof. The relation $\Delta\left(\alpha_{0}, \ldots, \alpha_{n-1}\right)$ is generated by tuples cube ${ }_{i}^{n}(a, b)$ for $a \equiv_{\alpha_{i}} b$. So, $\Delta\left(\alpha_{0}, \ldots, \alpha_{n-1}\right)$ is the set of all tuples of the form

$$
t\left(\operatorname{cube}_{i_{0}}^{n}\left(a_{0}, b_{0}\right), \ldots, \operatorname{cube}_{i_{k-1}}^{n}\left(a_{k-1}, b_{k-1}\right)\right)
$$

where $t \in \operatorname{Clo}_{k} \mathbf{A}$, and for all $j<k$ we have $i_{j}<n, a_{j} \equiv b_{j}$ modulo $\alpha_{i_{j}}$. The description in the statement of the lemma can be obtained from this by grouping together $\operatorname{cube}_{i_{j}}^{n}\left(a_{j}, b_{j}\right)$ 's with the same index $i_{j}$, and applying the term $t$ coordinatewise.

Example. In the ternary commutator case, the lemma tells that

$$
\begin{gathered}
\Delta\left(\alpha_{0}, \ldots, \alpha_{n-1}\right)=\left\{\left(t\left(\mathbf{a}_{0}, \mathbf{a}_{1}, \mathbf{a}_{2}\right), t\left(\mathbf{b}_{0}, \mathbf{a}_{1}, \mathbf{a}_{2}\right), t\left(\mathbf{a}_{0}, \mathbf{b}_{1}, \mathbf{a}_{2}\right), t\left(\mathbf{b}_{0}, \mathbf{b}_{1}, \mathbf{a}_{2}\right),\right.\right. \\
\left.t\left(\mathbf{a}_{0}, \mathbf{a}_{1}, \mathbf{b}_{2}\right), t\left(\mathbf{b}_{0}, \mathbf{a}_{1}, \mathbf{b}_{2}\right), t\left(\mathbf{a}_{0}, \mathbf{b}_{1}, \mathbf{b}_{2}\right), t\left(\mathbf{b}_{0}, \mathbf{b}_{1}, \mathbf{b}_{2}\right)\right) \mid \\
\left.m_{0}, m_{1}, m_{2} \in \mathbb{N}_{0}, t \in \mathrm{Clo}_{m_{0}+m_{1}+m_{2}} \mathbf{A}, \forall i<3: \mathbf{a}_{i}, \mathbf{b}_{i} \in A^{m_{i}}, \mathbf{a}_{i} \equiv_{\alpha_{i}} \mathbf{b}_{i}\right\} .
\end{gathered}
$$

The graphical representation of a typical element of this relation is given in Figure2. 


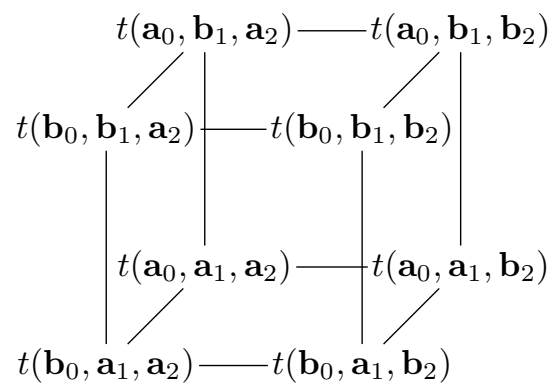

Figure 2.

Lemma 3.3. Let $\mathbf{A}$ be an algebra, and $\alpha_{0}, \ldots, \alpha_{n-1}, \gamma \in$ Con $\mathbf{A}$. Then $\alpha_{0}, \ldots, \alpha_{n-2}$ centralize $\alpha_{n-1}$ modulo $\gamma$ if for every tuple

$$
\left(a_{0 \ldots 0}, \ldots, a_{1 \ldots 1}, b_{0 \ldots 0}, \ldots, b_{1 \ldots 1}\right) \in \Delta\left(\alpha_{0}, \ldots, \alpha_{n-1}\right)
$$

such that $a_{\mathbf{k}} \equiv_{\gamma} b_{\mathbf{k}}$ for all $\mathbf{k} \in 2^{n-1} \backslash\{1 \ldots 1\}$ we have also $a_{1 \ldots 1} \equiv_{\gamma} b_{1 \ldots 1}$.

Lemma 3.4. Let $\mathbf{A}$ be an algebra with congruences $\alpha_{0}, \ldots, \alpha_{n}, i<n$, and $s_{i}: k \mapsto$ $k_{(0)} \ldots k_{(i-1)} \overline{k_{(i)}} k_{(i+1)} \ldots k_{(n-1)}$. Then

(i) if $d \in\{0,1\}$ and $\mathbf{a} \in \Delta\left(\alpha_{0}, \ldots, \alpha_{n-1}\right)$ then

$$
\text { face }_{i}^{d} \mathbf{a} \in \Delta\left(\alpha_{0}, \ldots, \alpha_{i-1}, \alpha_{i+1}, \ldots, \alpha_{n-1}\right) ;
$$

(ii) if $\mathbf{a} \in \mathbf{A}^{2^{n-1}}$ such that

$$
\text { face }_{i}^{0} \mathbf{a}=\text { face }_{i}^{1} \mathbf{a} \in \Delta\left(\alpha_{0}, \ldots, \alpha_{i-1}, \alpha_{i+1}, \ldots, \alpha_{n-1}\right),
$$

then $\mathbf{a} \in \Delta\left(\alpha_{0}, \ldots, \alpha_{n-1}\right)$;

(iii) $\Delta\left(\alpha_{0}, \ldots, \alpha_{n-1}\right)^{s_{i}}=\Delta\left(\alpha_{0}, \ldots, \alpha_{n-1}\right)$.

Furthermore, if $\mathbf{A}$ is a Mal'cev algebra then the binary relation

$$
\delta=\left\{\left(\text { face }_{i}^{0} \mathbf{a}, \text { face }_{i}^{1} \mathbf{a}\right) \mid \mathbf{a} \in \Delta\left(\alpha_{0}, \ldots, \alpha_{n-1}\right)\right\}
$$

is a congruence on $\Delta\left(\alpha_{0}, \ldots, \alpha_{i-1}, \alpha_{i+1}, \ldots, \alpha_{n}\right)$.

Proof. Since all the considered relations are compatible, we can check the validity of particular inclusions on the generators of the relations, i.e., tuples cube ${ }_{i, n}(a, b)$. In detail, to prove (i) one has to observe that

$$
\text { face }_{i}^{d} \operatorname{cube}_{j}^{n}(a, b)= \begin{cases}\operatorname{cube}_{j}^{n-1}(a, b) & \text { if } j<i, \\ (c, \ldots, c) & \text { where } c \in\{a, b\} \text { if } j=i, \\ \operatorname{cube}_{j-1}^{n-1}(a, b) & \text { if } j>i,\end{cases}
$$

and consequently

$$
\begin{aligned}
\operatorname{face}_{i}^{d}\left\{\operatorname{cube}_{j}^{n}(a, b) \mid j<n, a \equiv_{\alpha_{j}} b\right\} \\
\quad=\left\{\operatorname{cube}_{j}^{n-1}(a, b) \mid j<i, a \equiv_{\alpha_{j}} b\right\} \cup\left\{\operatorname{cube}_{j-1}^{n-1}(a, b) \mid i<j<n, a \equiv_{\alpha_{j}} b\right\} .
\end{aligned}
$$

Hence the relation generated by the left hand side is the same as the relation generated by the right hand side which gives the desired equality.

For (ii), first observe that

$$
\left\{\mathbf{a} \mid \text { face }_{i}^{0} \mathbf{a}=\text { face }_{i}^{1} \mathbf{a} \in \Delta\left(\alpha_{0}, \ldots, \alpha_{i-1}, \alpha_{i+1}, \ldots, \alpha_{n-1}\right)\right\}
$$


is the $2^{n}$-ary relation generated by tuples $\mathbf{a}$ such that face ${ }_{i}^{0} \mathbf{a}=$ face $_{i}^{1} \mathbf{a}=\operatorname{cube}_{j, n-1}(a, b)$, $a \equiv b$ modulo $\alpha_{j}$, or $\alpha_{j+1}$ when $j<i$, or $j \geq i$ respectively. Second, if a is such a tuple then $\mathbf{a}=\operatorname{cube}_{j}^{n}(a, b)$ if $j<i$, or $\mathbf{a}=\operatorname{cube}_{j+1}^{n}(a, b)$ if $j \geq i$. In either case, $\mathbf{a} \in \Delta\left(\alpha_{0}, \ldots, \alpha_{n-1}\right)$ for $a, b$ that are congruent modulo the corresponding congruence.

The item (iii) is a consequence of the fact that

$$
\left(\operatorname{cube}_{j}^{n}(a, b)\right)^{s_{i}}= \begin{cases}\operatorname{cube}_{j}^{n}(b, a) & \text { if } j=i, \text { or } \\ \operatorname{cube}_{j}^{n}(a, b) & \text { otherwise. }\end{cases}
$$

Hence $\left(\operatorname{cube}_{j}^{n} \alpha_{j}\right)^{s_{i}}=$ cube $_{j}^{n} \alpha_{j}$ from the symmetry of congruences. From the definition of $\Delta\left(\alpha_{0}, \ldots, \alpha_{n-1}\right)$, we get that

$$
\Delta\left(\alpha_{0}, \ldots, \alpha_{n-1}\right)^{s_{i}}=\Delta\left(\alpha_{0}, \ldots, \alpha_{n-1}\right) .
$$

From items (i)-(iii) we already know that the binary relation $\delta$ is a reflexive symmetric binary relation on $\Delta\left(\alpha_{0}, \ldots, \alpha_{i-1}, \alpha_{i+1}, \ldots, \alpha_{n-1}\right)$. The rest follows from Lemma 2.2 .

Lemma 3.5. Let A be a Mal'cev algebra with congruences $\alpha_{0}, \ldots, \alpha_{n-1}$. Then $\psi_{\mathbf{j}}\left(\Delta\left(\alpha_{0}, \ldots, \alpha_{n-1}\right)\right)=\psi_{\mathbf{k}}\left(\Delta\left(\alpha_{0}, \ldots, \alpha_{n-1}\right)\right)$ for all $\mathbf{j}, \mathbf{k} \in 2^{n}$.

Proof. For simplicity, let $\psi_{\mathbf{k}}=\psi_{\mathbf{k}}\left(\Delta\left(\alpha_{0}, \ldots, \alpha_{n-1}\right)\right)$. If $s_{i}$ is the permutation on $2^{n}$ defined by $s_{i}(\mathbf{k})=k_{0} \ldots k_{i-1} \overline{k_{i}} k_{i+1} \ldots k_{n-1}$ then from Lemma3.4(iii), we know that $\Delta\left(\alpha_{0}, \ldots, \alpha_{n-1}\right)=\Delta\left(\alpha_{0}, \ldots, \alpha_{n-1}\right)^{s_{i}}$. This gives, by Lemma 2.1 that $\psi_{\mathbf{k}}=\psi_{s_{i}(\mathbf{k})}$ for all $i<n$. But, if $i_{0}, \ldots, i_{m-1}$ are exactly those indices $i$ such that $k_{i} \neq j_{i}$ then $\mathbf{j}=s_{i_{0}} \circ \cdots \circ s_{i_{m-1}}(\mathbf{k})$, and consequently $\psi_{\mathbf{k}}=\psi_{\mathbf{j}}$ for all $\mathbf{j}, \mathbf{k}$.

Instead of proving Theorem 1.2 directly, we will prove the following refinement. The theorem is then given by equivalence of (1) and (4).

Proposition 3.6. If $\mathbf{A}$ is a Mal'cev algebra, $\alpha_{0}, \ldots, \alpha_{n-1} \in \operatorname{Con} \mathbf{A}$, and $a, b \in \mathbf{A}$; then the following is equivalent

(1) $(a, b) \in \psi_{1 \ldots 1}\left(\Delta\left(\alpha_{0}, \ldots, \alpha_{n-1}\right)\right)$;

(2) $(a, \ldots, a, b) \in \Delta\left(\alpha_{0}, \ldots, \alpha_{n-1}\right)$;

(3) there exists $c_{0}, \ldots, c_{2^{n-1}-2}$ such that

$$
\left(c_{0}, \ldots, c_{2^{n-1}-2}, a, c_{0}, \ldots, c_{2^{n-1}-2}, b\right) \in \Delta\left(\alpha_{0}, \ldots, \alpha_{n-1}\right) .
$$

(4) $a \equiv{ }_{\left[\alpha_{0}, \ldots, \alpha_{n-1}\right]} b$;

Proof. The implication $(1) \rightarrow(2)$ is direct corollary of Lemma 2.3. given that $\Delta\left(\alpha_{0}, \ldots, \alpha_{n-1}\right)$ contains all constant tuples, in particular $(a, \ldots, a) .(2) \rightarrow(3)$ is trivial. For $(3) \rightarrow(4)$ suppose that $(3)$ is satisfied for a pair $(a, b)$ then since $c_{i} \equiv c_{i} \bmod \left[\alpha_{0}, \ldots, \alpha_{n-1}\right]$ for all $i<2^{n-1}-1$ and $\alpha_{0}, \ldots, \alpha_{n-2}$ centralize $\alpha_{n-1}$ modulo $\left[\alpha_{0}, \ldots, \alpha_{n-1}\right]$, we have $a \equiv b \bmod \left[\alpha_{0}, \ldots, \alpha_{n-1}\right]$ from Lemma 3.3 .

The last to prove is $(4) \rightarrow(1)$, in other words that

$$
\left[\alpha_{0}, \ldots, \alpha_{n-1}\right] \leq \psi_{1 \ldots 1}\left(\Delta\left(\alpha_{0}, \ldots, \alpha_{n-1}\right)\right) .
$$

Let $\psi=\psi_{1 \ldots 1}\left(\Delta\left(\alpha_{0}, \ldots, \alpha_{n-1}\right)\right)$; from Lemma 3.5 we know that

$$
\psi=\psi_{1 \ldots 1}\left(\Delta\left(\alpha_{0}, \ldots, \alpha_{n-1}\right)\right)=\psi_{\mathbf{k}}\left(\Delta\left(\alpha_{0}, \ldots, \alpha_{n-1}\right)\right)
$$

for all $\mathbf{k} \in 2^{n}$, so we do not have to distinguish between forks at different coordinates. To prove (3.1) by the definition of the commutator, it is enough to prove that 
$\alpha_{0}, \ldots, \alpha_{n-2}$ centralize $\alpha_{n-1}$ modulo $\psi$. For that we will use Lemma 3.3. Suppose that

$$
\left(a_{0 \ldots 0}, \ldots, a_{1 \ldots 1}, b_{0 \ldots 0}, \ldots, b_{1 \ldots 1}\right) \in \Delta\left(\alpha_{0}, \ldots, \alpha_{n-1}\right),
$$

and $a_{\mathbf{i}} \equiv_{\psi} b_{\mathbf{i}}$ for all $\mathbf{i} \in 2^{n-1} \backslash\{1 \ldots 1\}$. By repeated use of Lemma 2.3 we can replace $b_{00 \ldots 0}, \ldots, b_{01 \ldots 1}$ by the respective $a_{\mathbf{i}}$ 's. Hence,

$$
\left(a_{00 \ldots 0}, \ldots, a_{01 \ldots 1}, a_{11 \ldots 1}, a_{00 \ldots 0}, \ldots, a_{01 \ldots 1}, b_{11 \ldots 1}\right) \in \Delta\left(\alpha_{0}, \ldots, \alpha_{n-1}\right) .
$$

In addition, we know from Lemma 3.4(i) and 3.4(ii) that

$$
\left(a_{00 \ldots 0}, \ldots, a_{01 \ldots 1}, a_{11 \ldots 1}, a_{00 \ldots 0}, \ldots, a_{01 \ldots 1}, a_{11 \ldots 1}\right) \in \Delta\left(\alpha_{0}, \ldots, \alpha_{n-1}\right) .
$$

So, $a_{11 \ldots 1} \equiv_{\psi} b_{11 \ldots 1}$ which concludes the proof that $\alpha_{0}, \ldots, \alpha_{n-2}$ centralize $\alpha_{n-1}$ modulo $\psi$.

In the last proposition some parts have been already known. The proposition (in the case of Mal'cev algebras) generalize Theorem 4.9 of [8] which gives equivalence of (2), (3), and (4) for binary commutators in congruence modular varieties. The omitted equivalence of (1) and (3) in the binary case can be easily derived from the known fact that $\Delta\left(\alpha_{0}, \alpha_{1}\right)$ is a congruence on rows. Furthermore, for the higher commutators, the implication $(3) \rightarrow(4)$ for the variety of groups has appeared in [13]; and if all $\alpha_{i}$ 's are principal congruences, the equivalence of (2) and (4) is given by [2, Lemma 6.13] (via an easy translation similar to Lemma 3.2).

\section{Strong Cube terms, And Clones of operations PRESERVInG COMMUTATORS}

We will use terms that generalize Mal'cev terms. These terms will play similar role as a difference term in the case of binary commutator. A $\left(2^{n}-1\right)$-ary term $q_{n}$ is a strong $n$-cube term if it satisfies

$$
q_{n}\left(x_{00 \ldots 0}, x_{10 \ldots 0}, \ldots, x_{01 \ldots 1}\right) \approx x_{11 \ldots 1}
$$

whenever there is $i<n$ such that for all $\mathbf{k}$ we have $x_{\mathbf{k}}=x_{k_{0} \ldots k_{i-1}} \overline{k_{i}} k_{i+1} \ldots k_{n-1}$. This gives a set of $n$ identities, each with $2^{n-1}$ variables. The two identities for strong 2-cube term are

$$
q_{2}(x, y, x) \approx y \quad \text { and } \quad q_{2}(x, x, y) \approx y .
$$

So, the term $q_{2}(y, x, z)$ is a Mal'cev term, and if $q$ is a Mal'cev term then $q(y, x, z)$ is a strong 2-cube term. The three identities for strong 3 -cube term are

$$
\begin{aligned}
& q_{3}(x, y, z, w, x, y, z) \approx w \\
& q_{3}(x, y, x, y, z, w, z) \approx w \\
& q_{3}(x, x, y, y, z, z, w) \approx w .
\end{aligned}
$$

Strong cube terms are stricter version of cube terms introduced in 4$]$; a $\left(2^{n}-1\right)$ ary term is an $n$-cube term if it satisfies

$$
q_{n}\left(x_{00 \ldots 0}, x_{10 \ldots 0}, \ldots, x_{01 \ldots 1}\right) \approx x_{11 \ldots 1}
$$

whenever there is $i<n$ such that $x_{\mathbf{k}}=x_{\mathbf{l}}$ for all $\mathbf{k}, \mathbf{l}$ such that $k_{i}=l_{i}$. This gives $n$ two-variable identities compared to the $2^{n-1}$ variables used in the identities for strong cube terms. One can see that for $n \geq 2$ every strong $n$-cube term satisfies the identities of an $n$-cube term; the $i$-th identity of a cube term is implied by almost any identity for a strong cube term except for the $i$-th one.

Lemma 4.1. The following is equivalent for any algebra $\mathbf{A}$. 
(1) A has a strong $n$-cube term for all $n \geq 2$,

(2) A has a strong $n$-cube term for some $n \geq 2$,

(3) A has a Mal'cev term.

Proof. From the observation in the above paragraphs, we know that the condition (3) is equivalent to

(3') A has a strong 2-cube term.

We will prove equivalence of (1), (2), and (3'). The implication (1) $\rightarrow(2)$ is trivial. For $(2) \rightarrow\left(3^{\prime}\right)$ observe that if $q_{n}$ is a strong $n$-cube term then $q_{2}(x, y, z)=$ $q_{n}(x, \ldots, x, x, y, z)$ is a strong 2 -cube term. For the last implication $\left(3^{\prime}\right) \rightarrow(1)$ we can construct strong cube terms by the recursion:

$$
q_{n+1}\left(x_{0}, \ldots, x_{2^{n+1}-1}\right)=q_{2}\left(q_{n}\left(x_{0}, \ldots, x_{2^{n}-2}\right), x_{2^{n}-1}, q_{n}\left(x_{2^{n}}, \ldots, x_{2^{n+1}-2}\right)\right)
$$

It is easy to check that if $q_{n}$ is a strong $n$-cube term and $q_{2}$ is a strong 2-cube term then $q_{n+1}$ is a strong $(n+1)$-cube term.

The following lemma is the key for proving Theorem 1.3 and a lot of properties of higher commutators in Mal'cev varieties.

Lemma 4.2. Let $\mathbf{A}$ be an algebra with a strong $n$-cube term $q_{n}, \alpha_{1}, \ldots, \alpha_{n} \in$ Con $\mathbf{A}$. Then $\mathbf{a} \in \Delta\left(\alpha_{1}, \ldots, \alpha_{n}\right)$ if and only if

$$
\text { face }_{i}^{0} \mathbf{a} \in \Delta\left(\alpha_{0}, \ldots, \alpha_{i-1}, \alpha_{i+1}, \ldots, \alpha_{n-1}\right)
$$

for each $i$ and $q_{n}\left(a_{00 \ldots} \ldots, a_{10 \ldots 0}, \ldots, a_{01 \ldots 1}\right) \equiv_{\left[\alpha_{0}, \ldots, \alpha_{n-1}\right]} a_{11 \ldots 1}$.

Proof. We will prove the lemma in two steps.

Claim 1. If face ${ }_{i}^{0} \mathbf{a} \in \Delta\left(\alpha_{0}, \ldots, \alpha_{i-1}, \alpha_{i+1}, \ldots, \alpha_{n-1}\right)$ for each $i<n$, and $q_{n}\left(a_{00 \ldots 0}\right.$, $\left.a_{10 \ldots 0}, \ldots, a_{01 \ldots 1}\right)=a_{11 \ldots 1}$ then $\mathbf{a} \in \Delta\left(\alpha_{1}, \ldots, \alpha_{n-1}\right)$;

For $\mathbf{k} \in 2^{n}$ and $\mathbf{b} \in A^{2^{n}}$ let $\mathbf{a}^{\mathbf{k}}$ denotes the $2^{n}$-tuple

$$
\mathbf{a}^{\mathbf{k}}=\left(a_{00 \ldots 0}, a_{k_{0} 0 \ldots 0}, a_{0 k_{1} \ldots 0}, \ldots, a_{k_{0} k_{1} \ldots k_{n-1}}\right) \text {. }
$$

Note that if $k_{i}=0$ then face ${ }_{i}^{0} \mathbf{a}^{\mathbf{k}}=$ face $_{i}^{1} \mathbf{a}^{\mathbf{k}}=$ face $_{i}^{0} \mathbf{a}^{k_{0} \ldots k_{i-1} 1 k_{i+1} \ldots k_{n-1}}$. Suppose that the tuple a satisfies the premise of the claim. The fact that $\mathbf{a}^{\mathbf{k}} \in$ $\Delta\left(\alpha_{0}, \ldots, \alpha_{n-1}\right)$ for all $\mathbf{k} \neq 11 \ldots 1$ follows from induction on the number of 0 's in $\mathbf{k}$ - the base step is given by Lemma 3.4(ii) and the assumption; the induction step is given by Lemma 3.4(i) and (ii) and the above observation. Next we claim that

$$
q_{n}^{\mathbf{A}^{2^{n}}}\left(\mathbf{a}^{00 \ldots 0}, \ldots, \mathbf{a}^{01 \ldots 1}\right)=\left(a_{00 \ldots 0}, \ldots, a_{01 \ldots 1}, q_{n}^{\mathbf{A}}\left(a_{00 \ldots 0}, \ldots, a_{01 \ldots 1}\right)\right) ;
$$

i.e., we have to show that

$$
q_{n}\left(a_{00 \ldots 0}, a_{j_{0} 0 \ldots 0}, \ldots, a_{0 j_{1} \ldots j_{n-1}}\right)=a_{\mathbf{j}}
$$

for every coordinate $\mathbf{j} \neq 11 \ldots 1$. The above is trivial for the coordinate $\mathbf{j}=11 \ldots 1$; for $\mathbf{j}$ with $j_{i}=0$ the identity follows from the $i$-th identity for a strong cube term. Finally, since the relation $\Delta\left(\alpha_{0}, \ldots, \alpha_{n-1}\right)$ is compatible with $q_{n}$ we know that the right hand side of (4.1) is in $\Delta\left(\alpha_{0}, \ldots, \alpha_{n-1}\right)$.

Claim 2. If $\mathbf{a} \in \Delta\left(\alpha_{0}, \ldots, \alpha_{n-1}\right)$ then $q_{n}\left(a_{00 \ldots 0}, \ldots, a_{01 \ldots 1}\right) \equiv_{\left[\alpha_{0}, \ldots, \alpha_{n-1}\right]} a_{11 \ldots 1}$. 
If $\left(a_{00 \ldots 0}, \ldots, a_{11 \ldots 1}\right) \in \Delta\left(\alpha_{0}, \ldots, \alpha_{n-1}\right)$ then from Lemma 3.4(i) we know that the tuple $\left(a_{00 \ldots 0}, \ldots, a_{01 \ldots 1}, q_{n}\left(a_{00 \ldots 0}, \ldots, a_{01 \ldots 1}\right)\right)$ satisfies the prerequisites of the first claim. Hence, we know that

$$
\left(a_{00 \ldots 0}, \ldots, a_{01 \ldots 1}, q_{n}\left(a_{00 \ldots 0}, \ldots, a_{01 \ldots 1}\right)\right) \in \Delta\left(\alpha_{0}, \ldots, \alpha_{n-1}\right)
$$

which shows that

$$
\left(a_{11 \ldots 1}, q_{n}\left(a_{00 \ldots 0}, \ldots, a_{01 \ldots 1}\right)\right) \in \psi_{11 \ldots 1}\left(\Delta\left(\alpha_{0}, \ldots, \alpha_{n-1}\right)\right)=\left[\alpha_{0}, \ldots, \alpha_{n-1}\right] .
$$

Finally, we get to the statement of this lemma. The 'only if' part is Claim 2 together with Lemma 3.4(i). For the 'if' part, if

$$
\text { face }_{i}^{0} \mathbf{a} \in \Delta\left(\alpha_{0}, \ldots, \alpha_{i-1}, \alpha_{i+1}, \ldots, \alpha_{n-1}\right)
$$

for all $i<n$, we know from Claim 1 that

$$
\left(a_{00 \ldots 0}, \ldots, a_{01 \ldots 1}, q_{n}\left(a_{00 \ldots 0}, \ldots, a_{01 \ldots 1}\right)\right) \in \Delta\left(\alpha_{0}, \ldots, \alpha_{n-1}\right) .
$$

From the last condition and Theorem 1.2, we know that $q_{n}\left(a_{00 \ldots 0}, \ldots, a_{01 \ldots 1}\right)$ and $a_{11 \ldots 1}$ are congruent modulo $\psi_{11 \ldots 1}\left(\Delta\left(\alpha_{0}, \ldots, \alpha_{n-1}\right)\right)$. Therefore, $\mathbf{a} \in \Delta\left(\alpha_{0}, \ldots, \alpha_{n-1}\right)$ from Lemma 2.3 .

In the rest of this chapter we use symbol $\left[\alpha_{0}, \ldots, \alpha_{n-1}\right]_{\mathbf{X}}$ to denote the commutator $\left[\alpha_{0}, \ldots, \alpha_{n-1}\right]$ computed in the algebra $\mathbf{X}$.

Corollary 4.3. Let A, B are algebras that share a universe, a Mal'cev operation, and congruences $\alpha_{0}, \ldots, \alpha_{n-1}$. Then

$$
\left[\alpha_{i_{0}}, \ldots, \alpha_{i_{k-1}}\right]_{\mathbf{A}}=\left[\alpha_{i_{0}}, \ldots, \alpha_{i_{k-1}}\right]_{\mathbf{B}}
$$

for all $k \leq n$ and $0 \leq i_{0}<\cdots<i_{k-1}<n$ if and only if $\Delta_{\mathbf{A}}\left(\alpha_{0}, \ldots, \alpha_{n-1}\right)=$ $\Delta_{\mathbf{B}}\left(\alpha_{0}, \ldots, \alpha_{n-1}\right)$.

Proof. We will prove the corollary by induction on $n$. The case $n=1$ is trivial; for the induction step suppose that for all congruences $\beta_{0}, \ldots, \beta_{n-1} \in$ Con $\mathbf{A} \cap$ Con $\mathbf{B}$ such that the commutators $\left[\beta_{i_{0}}, \ldots, \beta_{i_{k-1}}\right]$ agree in $\mathbf{A}$ and $\mathbf{B}$ for all $k<n$ and $i_{0}, \ldots, i_{k-1}$ pairwise distinct elements from $\{0, \ldots, n-1\}$, we have $\Delta_{\mathbf{A}}\left(\beta_{i_{0}}, \ldots, \beta_{i_{k-1}}\right)=$ $\Delta_{\mathbf{B}}\left(\beta_{i_{0}}, \ldots, \beta_{i_{k-1}}\right)$. In particular, we have $\Delta_{\mathbf{A}}\left(\alpha_{0}, \ldots, \alpha_{i-1}, \alpha_{i+1}, \ldots, \alpha_{n-1}\right)=$ $\Delta_{\mathbf{B}}\left(\alpha_{0}, \ldots, \alpha_{i-1}, \alpha_{i+1}, \ldots, \alpha_{n-1}\right)$ for all $i<n$. Let $q_{n}$ be a common strong $n$ cube operation of $\mathbf{A}$ and $\mathbf{B}$ (it can be derived from the common Mal'cev operation). From Lemma 4.2 we know that whenever $\mathbf{X}$ is a Mal'cev algebra then $\mathbf{a} \in \Delta_{\mathbf{X}}\left(\alpha_{0}, \ldots, \alpha_{n-1}\right)$ if and only if

$$
\begin{aligned}
& \text { face }_{j}^{0} \mathbf{a} \in \Delta_{\mathbf{X}}\left(\alpha_{0}, \ldots, \alpha_{i-1}, \alpha_{i+1}, \ldots, \alpha_{n-1}\right) \text { for all } j<n \text {, and } \\
& \qquad a_{2^{n-1}} \equiv_{\left[\alpha_{0}, \ldots, \alpha_{n-1}\right]_{\mathbf{x}}} q_{n}\left(a_{0}, \ldots, a_{2^{n}-2}\right) .
\end{aligned}
$$

Now, suppose that $\mathbf{a} \in \Delta_{\mathbf{A}}\left(\alpha_{0}, \ldots, \alpha_{n-1}\right)$ hence (4.2) is valid for $\mathbf{X}=\mathbf{A}$. But since the operation $q_{n}$, the commutators $\left[\alpha_{0}, \ldots, \alpha_{n-1}\right]$, and the relations $\Delta\left(\alpha_{0}, \ldots, \alpha_{i-1}\right.$, $\left.\alpha_{i+1}, \ldots, \alpha_{n-1}\right)$ agree in $\mathbf{A}$ and $\mathbf{B}$, we get that (4.2) is also true for $\mathbf{X}=\mathbf{B}$, hence $a \in \Delta_{\mathbf{B}}\left(\alpha_{0}, \ldots, \alpha_{n-1}\right)$. This shows that $\Delta_{\mathbf{A}}\left(\alpha_{0}, \ldots, \alpha_{n-1}\right) \subseteq \Delta_{\mathbf{B}}\left(\alpha_{0}, \ldots, \alpha_{n-1}\right)$. The other inclusion is analogous.

For the 'only if' part, suppose that $\Delta_{\mathbf{A}}\left(\alpha_{0}, \ldots, \alpha_{n-1}\right)=\Delta_{\mathbf{B}}\left(\alpha_{0}, \ldots, \alpha_{n-1}\right)$. From Lemma 3.4 (i) it follows that $\Delta_{\mathbf{A}}\left(\alpha_{i_{0}}, \ldots, \alpha_{i_{k-1}}\right)=\Delta_{\mathbf{B}}\left(\alpha_{i_{0}}, \ldots, \alpha_{i_{k-1}}\right)$ for all $\left\{i_{0}, \ldots, i_{k-1}\right\} \subseteq\{0, \ldots, n-1\}$ with $i$ 's pairwise distinct; and consequently from Theorem 1.2, $\left[\alpha_{i_{0}}, \ldots, \alpha_{i_{k-1}}\right]_{\mathbf{A}}=\left[\alpha_{i_{0}}, \ldots, \alpha_{i_{k-1}}\right]_{\mathbf{B}}$. 
Finally, we get to the proof of the Theorem 1.3. We restate the theorem once again.

Theorem 1.3. Let $\mathbf{A}$ be an algebra with Mal'cev term $q$, and $\alpha_{0}, \ldots, \alpha_{n-1}$ be congruences of $\mathbf{A}$. Then there exists a largest clone $\mathcal{C}$ on A containing $q$ such that it preserves congruences $\alpha_{0}, \ldots, \alpha_{n-1}$, and all commutators of the form $\left[\alpha_{i_{0}}, \ldots, \alpha_{i_{k}}\right]$ where $0 \leq i_{0}<\cdots<i_{k-1}<n$ agree in $\mathbf{A}$ and $(A, \mathcal{C})$.

Proof. We will show that the largest clone satisfying the required properties is the clone $\mathcal{C}$ of all polymorphisms of the relation $\Delta_{\mathbf{A}}\left(\alpha_{0}, \ldots, \alpha_{n-1}\right)$. Obviously $\mathcal{C} \supseteq$ Clo $\mathbf{A}$ which implies that $q \in \mathcal{C}$ and $\Delta_{\mathcal{C}}\left(\alpha_{0}, \ldots, \alpha_{n-1}\right) \supseteq \Delta_{\mathbf{A}}\left(\alpha_{0}, \ldots, \alpha_{n-1}\right)$. But since the relation $\Delta_{\mathbf{A}}\left(\alpha_{0}, \ldots, \alpha_{n-1}\right)$ is compatible with $\mathcal{C}$, and the relation $\Delta_{\mathcal{C}}\left(\alpha_{0}, \ldots, \alpha_{n-1}\right)$ is the smallest compatible relation containing $\operatorname{cube}_{i}^{n}(a, b)$ for all $a \equiv_{\alpha_{i}} b$ and $i<n$, we have $\Delta_{\mathcal{C}}\left(\alpha_{0}, \ldots, \alpha_{n-1}\right)=\Delta_{\mathbf{A}}\left(\alpha_{0}, \ldots, \alpha_{n-1}\right)$.

From Corollary 4.3, we know that $\mathcal{C}$ satisfies the specified property. The rest is to prove that $\mathcal{C}$ is the largest such clone. Let $\mathcal{B}$ be another clone satisfying the property. Then from the same corollary we get $\Delta_{\mathbf{A}}\left(\alpha_{0}, \ldots, \alpha_{n-1}\right)=\Delta_{\mathcal{B}}\left(\alpha_{0}, \ldots, \alpha_{n-1}\right)$, and consequently $\mathcal{B} \subseteq \operatorname{Pol}\left(\Delta_{\mathbf{A}}\left(\alpha_{0}, \ldots, \alpha_{n-1}\right)\right)=\mathcal{C}$.

\section{Proofs of Basic Properties of higher commutators}

In this chapter we will present alternative proofs of basic properties of higher commutators formulated in [5, 2. For an arbitrary algebra $\mathbf{A}$ and its congruences $\alpha_{0}, \ldots, \alpha_{n-1}, \beta_{0}, \ldots, \beta_{n-1}, \gamma$, and $\eta$ the following is satisfied

(HC1) $\left[\alpha_{0}, \ldots, \alpha_{n-1}\right] \leq \alpha_{0} \wedge \cdots \wedge \alpha_{n-1}$;

(HC2) if $\alpha_{i} \leq \beta_{i}$ for all $i$ then $\left[\alpha_{0}, \ldots, \alpha_{n-1}\right] \leq\left[\beta_{0}, \ldots, \beta_{n-1}\right]$;

(HC3) $\left[\alpha_{0}, \ldots, \alpha_{n-1}\right] \leq\left[\alpha_{1}, \ldots, \alpha_{n-1}\right]$.

Furthermore, if $\mathbf{A}$ is a Mal'cev algebra then

(HC4) if $\sigma$ is a permutation on the set $\{0, \ldots, n-1\}$ then

$$
\left[\alpha_{0}, \ldots, \alpha_{n-1}\right]=\left[\alpha_{\sigma(0)}, \ldots, \alpha_{\sigma(n-1)}\right]
$$

(HC5) congruences $\alpha_{0}, \ldots, \alpha_{n-2}$ centralize $\alpha_{n-1}$ modulo $\gamma$ if and only if

$$
\left[\alpha_{0}, \ldots, \alpha_{n-1}\right] \leq \gamma
$$

(HC6) if $\eta \leq \alpha_{0}, \ldots, \alpha_{n-1}$ then

$$
\left[\alpha_{0} / \eta, \ldots, \alpha_{n-1} / \eta\right]_{\mathbf{A} / \eta}=\left(\left[\alpha_{0}, \ldots, \alpha_{n-1}\right]_{\mathbf{A}} \vee \eta\right) / \eta
$$

(HC7) if $I$ is a non-empty set, and $\rho_{i}$ are congruences of $\mathbf{A}$ for all $i \in I$ then

$$
\bigvee_{i \in I}\left[\alpha_{0}, \ldots, \alpha_{n-2}, \rho_{i}\right]=\left[\alpha_{0}, \ldots, \alpha_{n-2}, \bigvee_{i \in I} \rho_{i}\right]
$$

(HC8) if $i=1, \ldots, n-1$ then $\left[\left[\alpha_{0}, \ldots, \alpha_{i-1}\right], \alpha_{i}, \ldots, \alpha_{n-1}\right] \leq\left[\alpha_{0}, \ldots, \alpha_{n-1}\right]$.

Although properties (HC1-3) can be derived directly from Theorem 1.2 and the definition of $\Delta\left(\alpha_{0}, \ldots, \alpha_{n-1}\right)$, we will omit these proofs because methods would work only for Mal'cev algebras; the general case have been proved in 11, Proposition 1.3]. We will prove properties ( $\mathrm{HC} 4),(\mathrm{HC} 5),(\mathrm{HC} 7)$, and (HC8) - the last property (HC6) is a corollary of (HC5).

Proposition 5.1 (HC4, 2, Proposition 6.1]). Let A be a Mal'cev algebra and let $\alpha_{0}, \ldots, \alpha_{n-1} \in$ Con $\mathbf{A}$. Then $\left[\alpha_{0}, \ldots, \alpha_{n-1}\right]=\left[\alpha_{\sigma(0)}, \ldots, \alpha_{\sigma(n-1)}\right]$ for each permutation $\sigma$ of $\{0, \ldots, n-1\}$. 
Proof. We claim that the relations $\Delta\left(\alpha_{\sigma(0)}, \ldots, \alpha_{\sigma(n-1)}\right)$ and $\Delta\left(\alpha_{0}, \ldots, \alpha_{n-1}\right)$ are identical up to permuting coordinates; precisely

$$
\Delta\left(\alpha_{\sigma(0)}, \ldots, \alpha_{\sigma(n-1)}\right)=\Delta\left(\alpha_{0}, \ldots, \alpha_{n-1}\right)^{\sigma^{\prime}}
$$

where $\sigma^{\prime}$ is defined by $\sigma^{\prime}(\mathbf{k})=k_{\sigma^{-1}(0)} \ldots k_{\sigma^{-1}(n-1)}$. One can check this fact by observing that $\sigma^{\prime}(\mathbf{k})(\sigma(i))=k_{i}$, and consequently

$$
\begin{aligned}
\Delta\left(\alpha_{\sigma(0)}, \ldots, \alpha_{\sigma(n-1)}\right)=\bigvee_{i<n} \operatorname{cube}_{i}^{n}\left(\alpha_{\sigma(i)}\right) & =\bigvee_{i<n}\left(\operatorname{cube}_{\sigma(i)}^{n}\left(\alpha_{\sigma(i)}\right)\right)^{\sigma^{\prime}} \\
= & \left(\bigvee_{i<n} \operatorname{cube}_{i, n}\left(\alpha_{i}\right)\right)^{\sigma^{\prime}}=\Delta\left(\alpha_{0}, \ldots, \alpha_{n-1}\right)^{\sigma^{\prime}} .
\end{aligned}
$$

Finally, $\sigma^{\prime}(11 \ldots 1)=11 \ldots 1$, so the statement is true from Theorem 1.2 and Lemma 2.1(ii).

Proposition 5.2 (HC5, 2, Lemma 6.2]). Let $\mathbf{A}$ be a Mal'cev algebra and $\alpha_{0}, \ldots, \alpha_{n-1}$, $\gamma$ be congruences of $\mathbf{A}$. Then $\alpha_{0}, \ldots, \alpha_{n-2}$ centralizes $\alpha_{n-1}$ modulo $\gamma$ if and only if $\gamma \geq\left[\alpha_{0}, \ldots, \alpha_{n-1}\right]$.

Proof. The 'only if' part is given by the definition of the commutator, to prove the 'if' part suppose that $\mathbf{a} \in \Delta\left(\alpha_{0}, \ldots, \alpha_{n-1}\right)$ such that $a_{k_{0} \ldots k_{n-2} 0} \equiv_{\gamma} a_{k_{0} \ldots k_{n-2} 1}$ for all $k \in 2^{n-1} \backslash\{1 \ldots 1\}$. We want to prove that $a_{11 \ldots 10} \equiv_{\gamma} a_{11 \ldots 1}$. By Lemma 4.2 we know that

$$
a_{11 \ldots 1} \equiv_{\left[\alpha_{0}, \ldots, \alpha_{n-1}\right]} q_{n}\left(a_{00 \ldots 0}, \ldots, a_{01 \ldots 1}\right)
$$

but the right hand side is modulo $\gamma$ congruent to

$$
q_{n}\left(a_{00 \ldots 0}, \ldots, a_{01 \ldots 10}, a_{1 \ldots 10}, a_{00 \ldots 0}, \ldots, a_{01 \ldots 10}\right)=a_{1 \ldots 10} .
$$

So, we know that $a_{1 \ldots 11} \equiv a_{1 \ldots 10}$ modulo $\gamma$ since $\gamma \geq\left[\alpha_{0}, \ldots, \alpha_{n-1}\right]$. And finally, $\alpha_{0}, \ldots, \alpha_{n-2}$ centralizes $\alpha_{n-1}$ modulo $\gamma$ from Lemma 3.3 .

The condition (HC6) is a direct corollary of condition (HC5); for a proof see 2 , Corollary 6.3]. The following two lemmata prepare for the proof of (HC7).

Lemma 5.3. Let $\mathbf{A}$ be an algebra, I a non-empty set, $\rho_{i} \in \mathrm{Con} \mathbf{A}$ for all $i \in I$, and $\alpha_{0}, \ldots, \alpha_{n-2} \in$ Con $\mathbf{A}$. Then

$$
\Delta\left(\alpha_{0}, \ldots, \alpha_{n-2}, \bigvee_{i \in I} \rho_{i}\right)=\bigvee_{i \in I} \Delta\left(\alpha_{0}, \ldots, \alpha_{n-2}, \rho_{i}\right) .
$$

Proof. The statement can be derived directly from the definition of the relation $\Delta\left(\alpha_{0}, \ldots, \alpha_{n-1}\right)$ by a simple calculation:

$$
\begin{aligned}
\Delta\left(\alpha_{0}, \ldots, \alpha_{n-2}, \bigvee_{i \in I} \rho_{i}\right) & =\left(\operatorname{cube}_{0}^{n} \alpha_{0} \vee \cdots \vee \operatorname{cube}_{n-2}^{n} \alpha_{n-2} \vee \operatorname{cube}_{n-1}^{n} \bigvee_{i \in I} \rho_{i}\right) \\
& =\bigvee_{i \in I}\left(\operatorname{cube}_{0}^{n} \alpha_{0} \vee \cdots \vee \operatorname{cube}_{n-2}^{n} \alpha_{n-2} \vee \operatorname{cube}_{n-1}^{n} \rho_{i}\right) \\
& =\bigvee_{i \in I} \Delta\left(\alpha_{0}, \ldots, \alpha_{n-2}, \rho_{i}\right) .
\end{aligned}
$$

The following lemma was in fact proved during the proof of [2, Lemma 6.4]. 
Lemma 5.4. Let $\mathbf{A}$ be an algebra, $I$ a non-empty set, $\rho_{i} \in$ Con $\mathbf{A}$ for all $i \in I$, and $\alpha_{0}, \ldots, \alpha_{n-2} \in$ Con $\mathbf{A}$. Then

$$
\left[\alpha_{0}, \ldots, \alpha_{n-2}, \bigvee_{i \in I} \rho_{i}\right]=\bigvee_{\substack{\left\{i_{0}, \ldots, i_{k-1}\right\} \subseteq I \\ k<\infty}}\left[\alpha_{0}, \ldots, \alpha_{n-2}, \bigvee_{i \in\left\{i_{0}, \ldots, i_{k-1}\right\}} \rho_{i}\right]
$$

Proof. Throughout the proof we will extensively use compactness of subuniverses of some fixed algebra that is if $\mathbf{A}_{i}$ for $i \in J$ are subalgebras of some algebra $\mathbf{A}$, and $a \in \bigvee_{i \in J} \mathbf{A}_{i}$ then there exists a finite set $K \subseteq J$ such that $a \in \bigvee_{i \in K} \mathbf{A}_{i}$. To shorten the notation let $\eta$ denotes the right hand side of the statement. Hence

$$
\eta=\bigvee_{\substack{\left\{i_{0}, \ldots, i_{k-1}\right\} \subseteq I, k<\infty}}\left[\alpha_{0}, \ldots, \alpha_{n-2}, \bigvee_{i \in\left\{i_{0}, \ldots, i_{k-1}\right\}} \rho_{i}\right] .
$$

First, we prove that $\alpha_{0}, \ldots, \alpha_{n-2}$ centralize $\bigvee_{i \in I} \rho_{i}$ modulo $\eta$.

Claim 1. If $\mathbf{a} \in \Delta\left(\alpha_{0}, \ldots, \alpha_{n-2}, \bigvee_{i \in I} \rho_{i}\right)$ then there is a finite set $S \subseteq I$ such that $\mathbf{a} \in \Delta\left(\alpha_{0}, \ldots, \alpha_{n-2}, \bigvee_{i \in S} \rho_{i}\right)$.

The claim follows from Lemma 5.3 and the note at the beginning of this proof.

Claim 2. If $a \equiv_{\eta} b$ then there is a finite set $T \subseteq I$ such that $a$ and $b$ are congruent modulo $\left[\alpha_{0}, \ldots, \alpha_{n-2}, \bigvee_{i \in T} \rho_{i}\right]$.

Again, there are finite sets $T_{0}, \ldots, T_{k-1}$ such that $a$ and $b$ are congruent modulo $\bigvee_{j=0}^{k-1}\left[\alpha_{0}, \ldots, \alpha_{n-2}, \bigvee_{i \in T_{j}} \rho_{i}\right]$. And,

$$
\bigvee_{j=0}^{k-1}\left[\alpha_{0}, \ldots, \alpha_{n-2}, \bigvee_{i \in T_{j}} \rho_{i}\right] \leq\left[\alpha_{0}, \ldots, \alpha_{n-2}, \bigvee_{\rho \in T} \rho\right]
$$

where $T=\bigcup_{j=0}^{k-1} T_{j}$. Which completes the proof of the second claim.

Suppose that $\mathbf{a} \in \Delta\left(\alpha_{0}, \ldots, \alpha_{n-2}, \bigvee_{i \in I} \rho_{i}\right)$ and $a_{k_{0} \ldots k_{n-2} 0} \equiv_{\eta} a_{k_{0} \ldots k_{n-2} 1}$ for all $\mathbf{k} \in 2^{n-1} \backslash\{1 \ldots 1\}$. Let $S$ be a finite set from Claim 1, and let $T_{\mathbf{k}}$ be finite sets such that $a_{k_{0} \ldots k_{n-2} 0}$ and $a_{k_{0} \ldots k_{n-2} 1}$ are congruent modulo $\left[\alpha_{0}, \ldots, \alpha_{n-2}, \bigvee_{\rho \in T_{\mathrm{k}}} \rho\right]$; such sets exist by Claim 2. Let $U=S \cup T_{0 \ldots 0} \cup \cdots \cup T_{1 \ldots 10}$ (note that $U$ is a finite set) and $\eta^{\prime}=\bigvee_{i \in U} \rho_{i}$. Then

(1) $\mathbf{a} \in \Delta\left(\alpha_{0}, \ldots, \alpha_{n-2}, \eta^{\prime}\right)$, and

(2) $a_{k_{0} \ldots k_{n-2} 0} \equiv_{\left[\alpha_{0}, \ldots, \alpha_{n-1}, \eta^{\prime}\right]} a_{k_{0} \ldots k_{n-2} 1}$ for all $k \in 2^{n-1} \backslash\{1 \ldots 1\}$.

So, from the Lemma 3.3 we know that $a_{1 \ldots 10} \equiv_{\left[\alpha_{0}, \ldots, \alpha_{n-1}, \eta^{\prime}\right]} a_{1 \ldots 11}$. Finally, $\left[\alpha_{0}, \ldots, \alpha_{n-1}, \eta^{\prime}\right] \leq$ $\left[\alpha_{0}, \ldots, \alpha_{n-1}, \eta\right]$ because $\eta^{\prime} \leq \eta$. Hence $\alpha_{0}, \ldots, \alpha_{n-2}$ centralize $\bigvee_{i \in I} \rho_{i}$ modulo $\eta$, and consequently $\left[\alpha_{0}, \ldots, \alpha_{n-2}, \bigvee_{i \in I} \rho_{i}\right] \leq \eta$.

The other inclusion is obvious from the fact that

$$
\left[\alpha_{0}, \ldots, \alpha_{n-2}, \bigvee_{i \in I} \rho_{i}\right] \geq\left[\alpha_{0}, \ldots, \alpha_{n-2}, \bigvee_{i \in J} \rho_{i}\right]
$$

for every finite set $J \subseteq I$.

Lemma 5.5 (2, Corollary 6.6]). Let $\mathbf{A}$ be a Mal'cev algebra and $\alpha_{1}, \ldots, \alpha_{n-1}$, $\rho_{1}, \ldots, \rho_{k}$ congruences of $\mathbf{A}$. Then

$$
\left[\alpha_{0}, \ldots, \alpha_{n-2}, \bigvee_{i=1}^{k} \rho_{i}\right]=\bigvee_{i=1}^{k}\left[\alpha_{0}, \ldots, \alpha_{n-2}, \rho_{i}\right]
$$


Proof. It suffices to prove the statement just for $k=2$. We will write

$$
\left(a_{0}, \ldots, a_{2^{n-1}-1}\right) \equiv_{\Delta\left(\alpha_{0}, \ldots, \alpha_{n-2}, \rho_{i}\right)}\left(b_{0}, \ldots, b_{2^{n-1}-1}\right)
$$

if $\left(a_{0}, \ldots, a_{2^{n-1}-1}, b_{0}, \ldots, b_{2^{n-1}-1}\right) \in \Delta\left(\alpha_{0}, \ldots, \alpha_{n-2}, \rho_{i}\right)$. Note that from Lemma 3.4, we know that the binary relation $\left\{(\mathbf{a}, \mathbf{b}): \mathbf{a} \equiv_{\Delta\left(\alpha_{0}, \ldots, \alpha_{n-2}, \rho_{i}\right)} \mathbf{b}\right\}$ is a congruence on $\Delta\left(\alpha_{0}, \ldots, \alpha_{n-2}\right)$. From Lemma 5.3, we know that

$$
\Delta\left(\alpha_{0}, \ldots, \alpha_{n-2}, \rho_{1} \vee \rho_{2}\right)=\Delta\left(\alpha_{0}, \ldots, \alpha_{n-2}, \rho_{1}\right) \vee \Delta\left(\alpha_{0}, \ldots, \alpha_{n-2}, \rho_{2}\right) .
$$

Since in Mal'cev algebras $\alpha \circ \beta=\operatorname{Sg}(\alpha \cup \beta)$ for any pair of congruences $\alpha$, $\beta$, we have that for all $\mathbf{a}, \mathbf{b} \in \Delta\left(\alpha_{0}, \ldots, \alpha_{n-2}\right), \mathbf{a} \equiv_{\Delta\left(\alpha_{0}, \ldots, \alpha_{n-2}, \rho_{1} \vee \rho_{2}\right)} \mathbf{b}$ if and only if there exists $\mathbf{c}$ such that $\mathbf{a} \equiv_{\Delta\left(\alpha_{0}, \ldots, \alpha_{n-2}, \rho_{1}\right)} \mathbf{c}$ and $\mathbf{b} \equiv_{\Delta\left(\alpha_{0}, \ldots, \alpha_{n-2}, \rho_{2}\right)} \mathbf{c}$.

Now, we prove that

$$
\left[\alpha_{0}, \ldots, \alpha_{n-2}, \rho_{1} \vee \rho_{2}\right] \leq\left[\alpha_{0}, \ldots, \alpha_{n-2}, \rho_{1}\right] \vee\left[\alpha_{0}, \ldots, \alpha_{n-2}, \rho_{2}\right] .
$$

Suppose that $a$ and $b$ are congruent modulo $\left[\alpha_{0}, \ldots, \alpha_{n-2}, \rho_{1} \vee \rho_{2}\right]$, hence from Proposition 3.6 there are $e_{0}, \ldots, e_{2^{n-1}-2}$ such that

$$
\left(e_{0}, \ldots, e_{2^{n-1}-2}, a\right) \equiv_{\Delta\left(\alpha_{0}, \ldots, \alpha_{n-2}, \rho_{1} \vee \rho_{2}\right)}\left(e_{0}, \ldots, e_{2^{n-1}-2}, b\right) .
$$

From the above observation, we know that there is a tuple $\mathbf{c} \in \Delta\left(\alpha_{0}, \ldots, \alpha_{n-2}\right)$ such that

$$
\mathbf{c} \equiv_{\Delta\left(\alpha_{0}, \ldots, \alpha_{n-2}, \rho_{1}\right)}\left(e_{0}, \ldots, e_{2^{n-1}-2}, a\right)
$$

and

$$
\mathbf{c} \equiv_{\Delta\left(\alpha_{0}, \ldots, \alpha_{n-2}, \rho_{2}\right)}\left(e_{0}, \ldots, e_{2^{n-1}-2}, b\right)
$$

If we use Lemma 4.2 for (5.2), we get that

$$
a \equiv{ }_{\left[\alpha_{0}, \ldots, \alpha_{n-2}, \rho_{1}\right]} q_{n}\left(c_{0}, \ldots, c_{2^{n-1}-1}, e_{0}, \ldots, e_{2^{n-1}-2}\right) ;
$$

similarly for (5.3), we get that

$$
b \equiv_{\left[\alpha_{0}, \ldots, \alpha_{n-2}, \rho_{2}\right]} q_{n}\left(c_{0}, \ldots, c_{2^{n-1}-1}, e_{0}, \ldots, e_{2^{n-1}-2}\right) .
$$

Altogether, $a$ and $b$ are congruent modulo $\left[\alpha_{0}, \ldots, \alpha_{n-2}, \rho_{1}\right] \vee\left[\alpha_{0}, \ldots, \alpha_{n-2}, \rho_{2}\right]$. Which completes the proof of (5.1). The other inclusion is given by (HC2).

Proposition 5.6 (HC7, [2, Lemma 6.7]). Let A be a Mal'cev algebra with congruences $\alpha_{0}, \ldots, \alpha_{n-2}$, and $\rho_{i}, i \in I$ for I non-empty set. Then

$$
\left[\alpha_{0}, \ldots, \alpha_{n-2}, \bigvee_{i \in I} \rho_{i}\right]=\bigvee_{i \in I}\left[\alpha_{0}, \ldots, \alpha_{n-2}, \rho_{i}\right]
$$

Proof. If $I$ is a finite set then the proposition is given by Lemma 5.5. If $I$ is infinite, we can first use Lemma 5.4 to get

$$
\left[\alpha_{0}, \ldots, \alpha_{n-2}, \bigvee_{i \in I} \rho_{i}\right]=\bigvee_{\left\{i_{0}, \ldots, i_{k-1}\right\} \subseteq I}\left[\alpha_{0}, \ldots, \alpha_{n-2}, \bigvee_{i \in\left\{i_{0}, \ldots, i_{k-1}\right\}} \rho_{i}\right]
$$

Then by using the finite case, the right hand side is equal to

$$
\bigvee_{\substack{\left\{i_{0}, \ldots, i_{k-1}\right\} \subseteq I, i \in\left\{i_{0}, \ldots, i_{k-1}\right\}}}\left[\alpha_{0}, \ldots, \alpha_{n-2}, \rho_{i}\right]=\bigvee_{i \in I}\left[\alpha_{0}, \ldots, \alpha_{n-2}, \rho_{i}\right]
$$

Proposition 5.7 (HC8, 2, Proposition 6.14]). Let A be a Mal'cev algebra with congruences $\alpha_{0}, \ldots, \alpha_{n-1}$, and $i \in\{1, \ldots, n-1\}$. Then

$$
\left[\left[\alpha_{0}, \ldots, \alpha_{i-1}\right], \alpha_{i}, \ldots, \alpha_{n-1}\right] \leq\left[\alpha_{0}, \ldots, \alpha_{n-1}\right] .
$$


Proof. For $m \geq i$, define the map $e_{m}: 2^{m} \rightarrow 2^{m-i+1}$ by $\mathbf{k} \mapsto k^{\prime} k_{i} \ldots k_{m-1}$ where $k^{\prime}=k_{0} \cdot k_{1} \cdot \ldots \cdot k_{i-1}$. We claim that

$$
\Delta\left(\left[\alpha_{0}, \ldots, \alpha_{i-1}\right], \alpha_{i}, \ldots, \alpha_{m-1}\right)^{e_{m}} \leq \Delta\left(\alpha_{0}, \ldots, \alpha_{m-1}\right) .
$$

Because $\Delta\left(\left[\alpha_{0}, \ldots, \alpha_{i-1}\right], \alpha_{i}, \ldots, \alpha_{m-1}\right)^{e_{m}}$ is clearly a subuniverse of $\mathbf{A}^{2^{m}}$ generated by the set

$$
\left(\operatorname{cube}_{0}^{m-i+1}\left[\alpha_{0}, \ldots, \alpha_{i-1}\right]\right)^{e_{m}} \cup \underset{j<m-i}{\bigcup}\left(\operatorname{cube}_{j+1}^{m-i+1} \alpha_{i+j}\right)^{e_{m}},
$$

it suffices to prove that this is a subset of $\Delta\left(\alpha_{0}, \ldots, \alpha_{m-1}\right)$. The inclusions

$$
\text { cube } \left._{j+1}^{m-i+1} \alpha_{i+j}\right)^{e_{m}} \subseteq \Delta\left(\alpha_{0}, \ldots, \alpha_{n-1}\right)
$$

are consequences of the fact that $e_{m}(\mathbf{k})(j+1)=k_{j+i}$ for all $j<m-i$. The other inclusion, (cube $\left.{ }_{0}^{m-i+1}\left[\alpha_{0}, \ldots, \alpha_{i-1}\right]\right)^{e_{m}} \subseteq \Delta\left(\alpha_{0}, \ldots, \alpha_{m-1}\right)$, can be proved by induction on $m$. If $m=i$ then

$$
\left(\operatorname{cube}_{0}^{1}\left[\alpha_{0}, \ldots, \alpha_{i-1}\right]\right)^{e_{m}}=\left\{(a, \ldots, a, b) \mid a \equiv_{\left[\alpha_{0}, \ldots, \alpha_{i-1}\right]} b\right\},
$$

and all the elements of this set are in $\Delta\left(\alpha_{0}, \ldots, \alpha_{i-1}\right)$ from Proposition 3.6 For the induction step, observe that

$$
\begin{aligned}
\operatorname{face}_{m}^{0}\left(\left(\operatorname{cube}_{0}^{(m+1)-i+1}(a, b)\right)^{e_{m+1}}\right)=\operatorname{face}_{m}^{1}\left(\left(\operatorname{cube}_{0}^{(m+1)-i+1}(a, b)\right)^{e_{m+1}}\right) \\
=\left(\operatorname{cube}_{0}^{m-i+1}(a, b)\right)^{e_{m}} .
\end{aligned}
$$

So the inclusion follows from Lemma 3.4(ii). Finally, from (5.4) for $m=n$, the fact that $e_{n}(\mathbf{k})=1 \ldots 1$ if and only if $\mathbf{k}=1 \ldots 1$, and Lemma 2.1 we get

$$
\psi_{1 \ldots 1}\left(\Delta\left(\left[\alpha_{0}, \ldots, \alpha_{i-1}\right], \alpha_{i}, \ldots, \alpha_{n-1}\right)\right) \leq \psi_{1 \ldots 1}\left(\Delta\left(\alpha_{0}, \ldots, \alpha_{n-1}\right)\right) .
$$

The rest is Theorem 1.2 .

Acknowledgements. The author would like to thank Erhard Aichinger for many hours of fruitful discussions and many helpful comments on the manuscript.

\section{REFERENCES}

[1] Erhard Aichinger, Peter Mayr, and Ralph McKenzie. On the number of finite algebraic structures. J. Eur. Math. Soc. (JEMS), 16(8):1673-1686, 2014.

[2] Erhard Aichinger and Nebojša Mudrinski. Some applications of higher commutators in Mal'cev algebras. Algebra Universalis, 63(4):367-403, 2010.

[3] Erhard Aichinger and Nebojša Mudrinski. On various concepts of nilpotence for expansions of groups. Publ. Math. Debrecen, 83(4):583-604, 2013.

[4] Joel Berman, Paweł Idziak, Petar Marković, Ralph McKenzie, Matthew Valeriote, and Ross Willard. Varieties with few subalgebras of powers. Trans. Amer. Math. Soc., 362(3):14451473, 2010.

[5] Andrei Bulatov. On the number of finite Mal'tsev algebras. In Contributions to general algebra, 13 (Velké Karlovice, 1999/Dresden, 2000), pages 41-54. Heyn, Klagenfurt, 2001.

[6] Andrei A. Bulatov. Three-element Mal'tsev algebras. Acta Sci. Math. (Szeged), 71(3-4):469$500,2005$.

[7] Andrei A. Bulatov and Paweł M. Idziak. Counting Mal'tsev clones on small sets. Discrete Math., 268(1-3):59-80, 2003.

[8] Ralph Freese and Ralph McKenzie. Commutator theory for congruence modular varieties, volume 125 of London Mathematical Society Lecture Note Series. Cambridge University Press, Cambridge, 1987.

[9] Martin Goldstern and Michael Pinsker. A survey of clones on infinite sets. Algebra Universalis, 59(3-4):365-403, 2008. 
[10] Paweł M. Idziak. Clones containing Mal'tsev operations. Internat. J. Algebra Comput., 9(2):213-226, 1999.

[11] Nebojša Mudrinski. On Polynomials in Mal'cev Algebras. PhD thesis, University of Novi Sad, 2009.

[12] B.A. Romov. Galois correspondence between iterative post algebras and relations on an infinite set. Cybernetics, 13(3):377-379, 1977.

[13] Jason Shaw. Commutator relations and the clones of finite groups. Algebra Universalis, $72(1): 29-52,2014$.

Department of Algebra, Faculty of Mathematics and Physics, Charles University in Prague, Sokolovská 83, 18675 Praha, Czech Republic

Institute for Algebra, Johannes Kepler University, 4040 Linz, Austria

E-mail address: oprsal@karlin.mff.cuni.cz 